\title{
Accessing Research Publications; A Brief Guideline on Literature Review
}

\author{
Dr. Md. Ashif Iqbal [1], Dr. Md. Huzzatul Islam Khan [2]
}

\section{Introduction:}

Idea always provokes a scientific research. And there is acritical distance that one needs to travel from having a research idea to arrive at research question. Researches always build up on past researches andcannotbe done in isolation. Only by learning about what has already been done, the past researches,we do cometo know about the scopes and opportunities for a new one. Otherwise, we may repeat something that's already been done, and this wouldn't be of interest to any scientific journal (if the goal is to try to replicate that study, intentionally repeating a previous study would beof interest).

So at the outset, one needs to find and to read published research papers to understand what's been done so far in that particular field of research. This is one of the mostcrucial preparatory steps and is often called "literature survey", "literature search", or "literature review". For a literature review, one needs to find relevant papersfirst.

\section{Searching and Accessing Research Papers:}

Research papers are found basically in two medium; print and electronic. For print medium you have to go through libraries, publishers, vendors and sometimes authors. It is time consuming and tiring process with print medium to use these for a review or survey. But electronic medium of journal is way more promising in systematic listing and sorting of desired papers for survey. Again, electronic medium is available everywhere and literally anywhere if you have a computer or any other devices with internet facilities throughout the whole universe. Now you don't have to move to door to doors anymore. These papers are stored in some databases or a journal's own website. You can search, read or discuss these papers through internet sitting on your couch from your home even on a vacation day and use them for your own research. All you need is a proper access to those stored papers from different databases or websites.

You can search for your desired papers free at most of the databases and websites. There are some freely available academic databases which anyone can use. Of these, Google Scholar (http://scholar.google.com) is among the most popular. Google Scholar indexes a wide range of journals across all academic fields, and it has the simplicity and power of the regular Google. PubMed (http://www.ncbi.nlm.nih.gov/pubmed) is another popular free database that indexes high quality journals in the health and life sciences. Medical researchers tend to use PubMed as a first-line resource, regardless of the availability of other databases. There are a number of databases that are available through subscription, such as Scopus and Web of Science, and these are sold to institutions. A detailed list (http://en.wikipedia.org/wiki/List_of_academic_databases_and_search_engines) of academic databases is available on Wikipedia.

Now, the question is how you are going to get access to those restricted research papers.

First option, you can subscribe for that paper or the journal or the database or the membership of the websites. They will definitely give you're a full access to your desired paper. This is online payment system where you will need an international VISA/Master/PayPal etc accounts or cards.

Second option, There are some special 
Vol. 7 No. 1 | April 2017

access provisions. Journal subscriptions can be expensive for universities. Thousands of journals around the world are using subscription models. Some universities have large budgets and they can subscribe to most or all of the journals that their researchers might need access to. In developing countries, universities often have limited budgets. But there's good news: some non-profit organizations and universities are working to address the problem of research access.INASP, the organization that has developed different e-learning courses, have an initiative aimed at improving research access. They work closely with librarians in some developing countries to help them negotiate with publishers for steeply discounted access to journal collections. Research4Life is another organisation working in the area of research access. They are well known for their access programs HINARI, AGORA, OARE, and ARDI. By participating in these programs, institutions in eligible developing countries can get free access to a vast amount of research literature. One might also be able to access resources available at other institutional libraries through inter-library loans. Visit inasp.info and research4life.org to learn more. And one can also speak with the librarian to find out if the institution works with any of these access programs or if there are any inter-library loan arrangements.

Sometimes one may find that he/she can access the full texts of some journal papers without logging in or without having to pay. This is of course very convenient! It may happen if:

-The paper is available because of open access. Either the journal is an open access journal, or this specific paper is in open access form.

-The paper is in a subscription journal but has access to this journal and signed in with the right credentials.

-One has accessed an archived copy of the paper on the author's personal website or elsewhere (for example, on a research sharing platform such as Mendeley or ResearchGate).
If anyone can read the full text of a paper, one might not think deeply about why he/she is able to read it. But it's good to know about open access and subscription journals, partly because one have to keep this distinction in mind when he/she is ready to select a journal for own paper.

\section{Using Academic Databases:}

To make the most of academic databases and obtain the most relevant papers, one need to think beyond typing key words to get results. Academic databases have many features that anybody can use to increase the effectiveness of the search. To learn about these features, one could look for tutorials on using a particular database.For example, a detailed tutorial on using Google Scholar is availablehere: http://scholar.google.com/intl/en/scholar/help.html and a tutorial on using PubMed is here: http://www.nlm.nih.gov/bsd/disted/pubmedtutorial/cover.html. Both the tutorials linked above are official tutorials, produced by the maintainers of the respective databases. They contain many useful tips on how to make the most of the databases, well beyond simple searching with key words. Finally, thearticle (http://googlescholar.blogspot.in/2014/10/using-google-scholar-in-scholarly.html) written by a leading biologist on using Google Scholar in scholarly workflows is so much helpful.

\section{Gathering the latest knowledge:}

Before the digital age, when journals were available mostly in the printed form, researchers would pick up an issue of a journal and go through it to look for articles of interest. Or they would browse periodicals that indexed the table of contents from the latest issues of select journals.Nowadays, you can access publications directly through academic databases that index thousands of journals! You don't have to go through one journal at a time. Still, you need to know the most well-established journals in someone's field. It's a good idea to browse these journals regularly whether online or in printed form - if there have access. This way, one would come to know about the cutting-edge work being carried out. This could be useful knowledge even if it's not directly related to someone own research agenda. 


\section{Sharing for betterment:}

This is something one could discuss with colleagues or on online networks such as the AuthorAID discussion list (http://dgroups.org/groups/authoraiddiscussion) and ResearchGate (http://researchgate.net/) or in the Linkedln(https://www.linkedin.com/) etc.

\section{Conclusion:}

A comprehensive review of the literature is important because it provides an up-to-date understanding of the subject and its significance to practice, identifies the methods used in previous research on the topic, helps to work out how to answer the questions - and indeed, what questions need to be asked, provides comparisons for one own research findings. This article will be helpful and could act as a preliminary guideline for accessing research publication for the new researcher.

\section{References:}

1. INASP Moodle, Accessing research publication, Ravi Murugesan, 2015 May. Available from: http://moodle.inasp.info/ 